\title{
Quantum Speed Limit for Perfect State Transfer in One Dimension
}

\author{
Man-Hong Yung* \\ Department of Physics, University of Illinois at Urbana-Champaign, Urbana IL 61801-3080, USA
}

(Dated: May 6, 2019)

\begin{abstract}
The basic idea of spin chain engineering for perfect quantum state transfer (QST) is to find a set of coupling constants in the Hamiltonian, such that a particular state initially encoded on one site will evolve freely to the opposite site without any dynamical controls. The minimal possible evolution time represents a speed limit for QST. We prove that the optimal solution is the one simulating the precession of a spin in a static magnetic field. We also argue that, at least for solidstate systems where interactions are local, it is more realistic to characterize the computation power by the couplings than the initial energy.
\end{abstract}

PACS numbers: 03.67.HK, 03.67.Lx, 03.67.-a

The transfer of a quantum state from one part of a physical unit e.g. qubit, to another part is a crucial ingredient for many quantum information processing protocols [1]. In fact, the ability to transmit "flying qubits" is known as one of the DiVincenzo's desiderata [2] for quantum communication. For long distance communication, it is natural to rely on optical means 3 . However, for systems involved in a sufficiently small scale where qubits can interact either directly or indirectly through other qubits, it seems to be more natural to exploit the interactions between them directly. In these scenarios, a typical assumption usually made is that the interactions between pairs of qubits can be arbitrarily switched on and off [4, [5]. In this way, a quantum state can be transmitted by a series of swap (SOS) operations. However, even in the absence of decoherence, the intrinsic problem of this protocol is that each swap would necessarily introduce some errors due to dynamical controls, the fidelity of the transferred states would therefore decay exponentially as the number of swaps increases. It is therefore of great practical interest to study schemes of perfect state transfer by free Hamiltonian evolution which is primarily designed to minimize dynamical controls as much as possible.

This work is also motivated by the question of the ultimate speed limit of solid-state quantum computers. The Margolus-Levitin theorem [6] suggests that the shortest time for a quantum state to evolve into an orthogonal state is limited by the initial energy. With this result, one can quantify the speed limit for elementary operations involving one or two qubits [7]. However, this theorem does not give an appropriate bound on the speed limit for the initial state to evolve into a particular state, for example the one dimensional quantum state transfer (QST) problem [cf. Eq. (2)]. The aim of this paper is to establish the speed limit for the QST problem. Following the scheme described in Ref. 11], one can generalize the study to more general quantum operations. In contrast with the Margolus-Levitin theorem, the quantum speed limit for QST will be characterized directly by the couplings in the Hamiltonian, instead of the initial energy.
As we shall see, this makes it not only experimentally more accessible, not also physically more reasonable.

Imperfect QST [8, 9] over a uniform spin chain was studied numerically, showing that perfect state transfer is impossible for long chains. However, it has been suggested 12] that perfect QST, without dynamical controls, is possible if we allow the couplings between the spins to be non-uniform. Moreover, it has also been pointed out 10] that there are many possible solutions for constructing spin chains which allow perfect state transfer, and the searching for the solutions is in general an inverse eigenvalue problem. Recently, there have been many quantum information processing proposals $[1,13,14,15,16,17,18,19,20,21]$ related to the concept of spin chain engineering. To boost the performance of the spin chains, or to evaluate the ultimate speed limit of these proposals, it is highly desirable to know how to engineer spin chains with optimal performance.

In the following, we shall show that the optimal solution for perfect state transfer is exactly the one proposed in Ref. 12, 22. Amusingly, this optimal set of couplings is the same as that describing spin precession in a static magnetic field. In discussing the one dimensional QST problem, one usually considers the Hamiltonian for the engineered spin- $1 / 2$ chains to be of the following form [23]:

$$
H=\sum_{j=1}^{N-1} \frac{\omega_{j}}{2}\left(\sigma_{j}^{x} \sigma_{j+1}^{x}+\sigma_{j}^{y} \sigma_{j+1}^{y}\right)+\sum_{j=1}^{N} \frac{\lambda_{j}}{2}\left(\sigma_{j}^{z}+1\right),
$$

where $\sigma_{j}$ 's are the standard Pauli matrices for the site $j$, and $N$ is the total number of sites in the spin chain. Both $\omega_{j}$ and $\lambda_{j}$ are real constants to be determined. We shall adopt the convention that $\left|0_{j}\right\rangle\left(\left|1_{j}\right\rangle\right)$ represents spin-down (spin-up) state.

In the quantum state transfer protocol, one site is encoded with a quantum state $\alpha|1\rangle+\beta|0\rangle$ representing the information of one qubit, while the rest are initialized to be spin-down. Define $U(\tau)=e^{-i H \tau}$, where $\tau$ is the evolution time and $\hbar=1$. Since $U(\tau)|000 \ldots 00\rangle=|000 \ldots 00\rangle$, 
the state is said to be perfectly transferred whenever

$$
U(\tau)|100 \ldots 00\rangle=e^{i \phi}|000 \ldots 01\rangle \quad,
$$

where $\phi$ is some known phase. Although the transferred state in general differs from the input state by a relative phase factor, i.e. $\alpha e^{i \phi}|1\rangle+\beta|0\rangle$, the phase factor could be corrected by some local operations and is thus usually ignored. To investigate further, it is clear that we can simply focus on the single-particle subspace $\{|\mathbf{1}\rangle \equiv|100 \ldots 0\rangle,|\mathbf{2}\rangle \equiv|010 \ldots 0\rangle, \ldots\}$, where $|\boldsymbol{x}\rangle$, $x=1,2,3, . ., N$ refers to a state with a single excitation at site $x$. In this subspace, the Hamiltonian in Eq. (10) is real, symmetrical and tridiagonal:

$$
H_{S}=\left(\begin{array}{ccccc}
\lambda_{1} & \omega_{1} & 0 & \cdots & 0 \\
\omega_{1} & \lambda_{2} & \omega_{2} & \cdots & 0 \\
0 & \omega_{2} & \lambda_{3} & \cdots & 0 \\
\vdots & \vdots & \vdots & \ddots & \omega_{N-1} \\
0 & 0 & 0 & \omega_{N-1} & \lambda_{N}
\end{array}\right)
$$

The eigenvalues, which must be non-degenerate 25], and the corresponding eigenvectors are denoted by $E_{k}$ and $\left|\boldsymbol{e}_{k}\right\rangle$ respectively. Let us consider not only transferring states from one end to the other but more generally the transition amplitude between the state $|\boldsymbol{x}\rangle$ and its mirrorinverted state $|\overline{\boldsymbol{x}}\rangle$, with $\bar{x} \equiv N-x+1$,

$$
\langle\overline{\boldsymbol{x}}|U(\tau)| \boldsymbol{x}\rangle=\sum_{k=1}^{N}\left\langle\overline{\boldsymbol{x}} \mid \boldsymbol{e}_{k}\right\rangle\left\langle\boldsymbol{e}_{k} \mid \boldsymbol{x}\right\rangle e^{-i E_{k} \tau} .
$$

Provided that the Hamiltonian in Eq. (11) or Eq. (3) is mirror symmetrical, i.e. $\lambda_{j}=\lambda_{\bar{j}}$ and $\omega_{j}=\omega_{N-j}$, one can show 10] that $\left\langle\overline{\boldsymbol{x}} \mid \boldsymbol{e}_{k}\right\rangle=(-1)^{k}\left\langle\boldsymbol{x} \mid \boldsymbol{e}_{k}\right\rangle$, and hence $|\langle\bar{x}|U(\tau)| x\rangle|=1$ if

$$
e^{-i E_{k} \tau}=(-1)^{k} e^{i \phi}
$$

The phase factor $e^{i \phi}$ here is exactly the same as that appeared in Eq. (2). It has been shown [10] that the condition in Eq. (5) is not only sufficient but also necessary for perfect state transfer in mirror symmetric chains. Given an eigenvalue spectrum $\left\{E_{k}\right\}$ satisfying Eq. (15), the corresponding coupling constants $\left\{\omega_{j}, \lambda_{j}\right\}$ can be uniquely determined. The task of solving for the solutions of $\left\{\omega_{j}, \lambda_{j}\right\}$ is therefore an inverse eigenvalue problem [24, 25], which generally has to be solved numerically. A spin chain is said to be engineered if its couplings are found in this way.

Perhaps the simplest solution to the Eq. (15) is the linear spectrum [12]. By mapping the states $|\boldsymbol{x}\rangle$ to be the eigenstates of the $J_{z}$ angular momentum operator, this solution also describes the precession of a spin $J=(N-1) / 2$ under a constant magnetic field pointing along the $x$-direction. Explicitly, the matrix elements of $H_{S}$ can be chosen as

$$
\lambda_{j}=0 \quad \text { and } \quad \omega_{j}=\frac{1}{2} \sqrt{j(N-j)} .
$$

TABLE I: Comparing the efficiency $\eta$ (defined in Eq. (7)) for various possible solutions to the problem of perfect state transfer through a spin chain of $N$ spins. For convenience, we have scaled the maximum tunneling matrix element $\omega_{\max }$ such that the minimal evolution time $\tau=\pi$ is fixed.

\begin{tabular}{lll}
\hline \hline Type of Spectrum & $\omega_{\max }$ & Efficiency $\eta$ \\
\hline Linear $^{a}$ & $\tilde{\omega}_{\max }$ & 1 \\
Quadratic $^{b}$ & $q(2 \alpha+N) \tilde{\omega}_{\max }$ & $q(2 \alpha+N)$ \\
Gapped linear I & $(1+4 q / N) \tilde{\omega}_{\max }$ & $(1+4 q / N)$ \\
Gapped linear II $^{d}$ & $q$ & $q$ \\
Cosine $^{e}$ & 108.5 & 14.0 \\
\hline \hline
\end{tabular}

${ }^{a}$ See Ref. 12]. $\tilde{\omega}_{\max }$ is defined in Eq. 8 and $\eta=1$ by definition.

${ }^{b}$ See Ref. 13]. For $N^{2} \gg 1, q(\alpha+1) \geq 1 / 2$ as required by $E_{1}-E_{0} \geq 1$, where $q$ is a positive integer and $\alpha$ is a rational number.

${ }^{c}$ See Ref. 15. For $N^{2} \gg 1$.

${ }^{d}$ See Ref. 10|. For $N=4$ only.

${ }^{e}$ See Ref. [20]. For $N=31$ (no analytic solution given).

The eigenvalue spectrum is linear in the sense that the energy spacing is uniform, $E_{k}=-(N-1) / 2+k-1, k=$ $1,2,3, \ldots, N$. From Eq. (4) and (5), perfect state transfer can be achieved for a time period of $\tau=\pi$.

Apparently, there are unlimited number of eigenvalue spectra satisfying the condition in Eq. (5). A number of particular solutions have also been found $10,13,15,20$. recently. To compare different sets of inter-qubit coupling constants $\left\{\omega_{j}\right\}$ generated from different energy eigenvalue spectra $\left\{E_{k}\right\}$, the efficiency $\eta$ can be quantified by the evolution time $\tau$ for completing the task of perfect state transfer, subject to the constraint that the maximum value of inter-spin coupling $\omega_{\max } \equiv \max \left\{\omega_{j}\right\}$ being normalized. Alternatively, one may fix the evolution time and compare the maximum coupling strength for different chains. Both cases can be properly captured by defining the efficiency as

$$
\eta=\frac{\omega_{\max } \tau}{\tilde{\omega}_{\max } \tilde{\tau}}
$$

where $\tilde{\omega}_{\max }$ and $\tilde{\tau}$ are respectively the maximum coupling and the evolution time of a reference spin chain, which we shall choose to be the one described in Eq. (6), i.e. $\tilde{\tau}=\pi$ and

$$
\tilde{\omega}_{\max }=\left\{\begin{array}{cc}
\frac{1}{4} \sqrt{N^{2}-1} & \text { for odd } N \\
\frac{1}{4} N & \text { for even } N .
\end{array}\right.
$$

With these definitions, our goal is to show that $\eta \geq 1$ for all engineered spin chains. This implies that the set of couplings in Eq. (6) is optimal and the quantum speed limit for one dimensional QST is established (i.e. given the same maximum couplings $\omega_{\max }=\tilde{\omega}_{\max }, \tau=\eta \tilde{\tau} \geq$ $\tilde{\tau})$. A comparison for different schemes of perfect state transfer is given in Table \

We start with the observation pointed out in Ref. [11]. Suppose the eigenvalues are ordered as $E_{1}>E_{2}>\cdots>$ 
$E_{N}$. Define $\Delta_{k} \equiv E_{k}-E_{k+1}$ and the range of the spectrum as $\Delta_{E} \equiv E_{1}-E_{N}=\sum_{k=1}^{N-1} \Delta_{k}$. From Eq. (5), the evolution time is limited by the minimum energy interval $\Delta_{\text {min }} \equiv \min \left\{\Delta_{k}\right\}, \tau \geq \pi / \Delta_{\min }$, while $\tilde{\tau}=\pi / \tilde{\Delta}_{\text {min }}$. From Eq. (7), we have

$$
\eta \geq \frac{\omega_{\max } \tilde{\Delta}_{\min }}{\tilde{\omega}_{\max } \Delta_{\min }}
$$

Suppose we scale the eigenvalue spectra such that $\tilde{\Delta}_{\text {min }}=$ $\Delta_{\min }$, then $\eta \geq 1$ if $\omega_{\max } \geq \tilde{\omega}_{\max }$. We shall show that it is indeed true.

In the single-spin subspace, applying the HellmannFeynman theorem to the Hamiltonian in Eq. (1), we have $\partial E_{k} / \partial \lambda_{j}=\frac{1}{2}\left\langle\boldsymbol{e}_{k}\left|\left(\sigma_{j}^{z}+1\right)\right| \boldsymbol{e}_{k}\right\rangle$ and $\partial E_{k} / \partial \omega_{j}=$ $2\left\langle\boldsymbol{e}_{k} \mid \boldsymbol{j}\right\rangle\left\langle\boldsymbol{j}+\mathbf{1} \mid \boldsymbol{e}_{k}\right\rangle$. It is clear that $\partial E_{k} / \partial \lambda_{j} \geq 0$. On the other hand, it is known 25] that the eigenvector $\left|\boldsymbol{e}_{k}\right\rangle$ has exactly $k-1$ sign changes in the position basis $\{|\boldsymbol{j}\rangle\}$. In other words, $\partial E_{1} / \partial \omega_{j} \geq 0$ and $\partial E_{N} / \partial \omega_{j} \leq 0$. Suppose we replace all $\omega_{j} \rightarrow \omega_{\max }$ and $\lambda_{j} \rightarrow \lambda_{\max }, \lambda_{\min }$ respectively, and invoke the solution of the eigenvalues for a uniformly coupled chain: $\lambda+2 \omega \cos \left(\frac{k \pi}{N+1}\right)$, we have

$$
\Delta_{E} \leq \Delta_{\lambda}+4 \omega_{\max }
$$

where $\Delta_{\lambda} \equiv \lambda_{\max }-\lambda_{\min }$ is the range of the spatial variations of the local potentials. Suppose, in the absence of, or with uniform, local potentials, we set $\Delta_{\lambda}=0$. Under the constraint that the evolution time to be the same, i.e. $\Delta_{\text {min }} \geq \tilde{\Delta}_{\text {min }} \equiv 1$, the range of any nonlinear spectrum must be at least greater then that of the linear spectrum by one, $\Delta_{E} \geq \tilde{\Delta}_{E}+1=N$, as the ratio between any two $\Delta_{k}$ 's must be a rational number [12]. One can then show from Eq. (8) and (10) that $\omega_{\max } \geq \tilde{\omega}_{\max }$. Of course, this argument is based on the assumption that the terms $\lambda_{j}$ play no role in the eigenvalue spectrum except a constant shift. The question is: could the speed limit be boosted, if spatially varying local potentials are allowed? The aim of the following paragraphs is to exclude such possibility, and at the same time provide a more rigorous proof.

Exploiting the symmetry of our problem, we can divide the $H_{S}$ in Eq. (3i) into two different subspaces [9, 10]. For even $N$, they are simply $\frac{1}{\sqrt{2}}\{|\boldsymbol{j}\rangle+|\overline{\boldsymbol{j}}\rangle\}$ and $\frac{1}{\sqrt{2}}\{|\boldsymbol{j}\rangle-|\overline{\boldsymbol{j}}\rangle\}, j=1,2, . ., N / 2$. The $N \times N$ Hamiltonian reduces into two $\frac{N}{2} \times \frac{N}{2}$ ones:

$$
\left(\begin{array}{ccccc}
\lambda_{1} & \omega_{1} & 0 & \ldots & 0 \\
\omega_{1} & \lambda_{2} & \omega_{2} & \ldots & 0 \\
0 & \omega_{2} & \lambda_{3} & \ldots & 0 \\
\vdots & \vdots & \vdots & \ddots & \omega_{N / 2-1} \\
0 & 0 & 0 & \omega_{N / 2-1} & \lambda_{N / 2} \pm \omega_{N / 2}
\end{array}\right)
$$

These two matrices are almost identical except one diagonal matrix element $\lambda_{N / 2} \pm \omega_{N / 2}$. Recall that $\left\langle\overline{\boldsymbol{x}} \mid \boldsymbol{e}_{k}\right\rangle=(-1)^{k}\left\langle\boldsymbol{x} \mid \boldsymbol{e}_{k}\right\rangle$, the eigenvectors of $H_{S}$ are automatically grouped into these two subspaces. In other words, the eigenvalue spectra of these two matrices are respectively $\nu_{k}=\left\{E_{1}, E_{3}, E_{5}, \ldots, E_{N-1}\right\}$ and $\mu_{k}=\left\{E_{2}, E_{4}, E_{6}, \ldots, E_{N}\right\}$. Consequently, the trace difference between the two matrices gives $\omega_{N / 2}=$ $(1 / 2) \sum_{k}\left(\nu_{k}-\mu_{k}\right)=(1 / 2) \sum_{k} \Delta_{2 k-1}$, which is minimized when all $\Delta_{2 k-1}=\tilde{\Delta}_{\text {min }}$, which corresponds to the case $\omega_{N / 2}=\tilde{\omega}_{\max }$. Hence we can conclude that $\omega_{\max } \geq \omega_{N / 2} \geq \tilde{\omega}_{\max }$ for even $N$.

For odd $N \geq 5$ (the case $N=3$ can be analytically solved separately), it becomes slightly more complicated. The subspaces are spanned by $\frac{1}{\sqrt{2}}\{|\boldsymbol{j}\rangle-|\overline{\boldsymbol{j}}\rangle\}$, and $\frac{1}{\sqrt{2}}\{|\boldsymbol{j}\rangle+|\overline{\boldsymbol{j}}\rangle\}$ together with $|\boldsymbol{m}\rangle$, where $j=$ $1,2,3, \ldots, m-1$ and $m \equiv(N+1) / 2$. The matrix formed by the latter is

$$
\left(\begin{array}{ccccc}
\lambda_{1} & \omega_{1} & 0 & 0 & 0 \\
\omega_{1} & \ddots & \vdots & \vdots & \vdots \\
0 & \cdots & \lambda_{m-2} & \omega_{m-1} & 0 \\
0 & \cdots & \omega_{m-1} & \lambda_{m-1} & \sqrt{2} \omega_{m} \\
0 & \cdots & 0 & \sqrt{2} \omega_{m} & \lambda_{m}
\end{array}\right)
$$

and the counterpart by the former can be obtained from the above matrix by removing the last row and the last column. Similar to the case of even $N$, the eigenvalue spectra are $\nu_{k}=\left\{E_{1}, E_{3}, \ldots, E_{N}\right\}$ and $\mu_{k}=$ $\left\{E_{2}, E_{4}, \ldots, E_{N-1}\right\}$ respectively. Our goal is still to show $\omega_{m} \geq \tilde{\omega}_{\max }$ when $\Delta_{\min } \geq \tilde{\Delta}_{\text {min }}$. To proceed, one can diagonalize the upper part of the matrix in Eq. (12), i.e. the part from $\lambda_{1}$ to $\lambda_{m-1}$, and the resulting matrix is of the bordered diagonal form (arrowhead matrix) 24]

$$
\left(\begin{array}{ccccc}
\lambda_{1} & 0 & \cdots & 0 & b_{1} \\
0 & \lambda_{2} & \cdots & 0 & b_{2} \\
\vdots & \vdots & \ddots & 0 & \vdots \\
0 & 0 & 0 & \lambda_{m-1} & b_{m-1} \\
b_{1} & b_{2} & \cdots & b_{m-1} & a
\end{array}\right)
$$

where $a=\lambda_{m}=\sum_{k=1}^{m} \nu_{k}-\sum_{k=1}^{m-1} \mu_{k}$ is the trace difference, and $b_{k}$ are the off-diagonal matrix elements satisfying the condition $2 \omega_{m}^{2}=\sum_{k=1}^{m-1} b_{k}^{2}$, as a result of of the diagonalization.

The characteristic polynomial $P(E)=\prod_{k=1}^{m}\left(E-\nu_{k}\right)$ of the matrix above can be written as

$$
P(E)=(E-a) Q(E)-\sum_{k=1}^{m-1} \frac{b_{k}^{2}}{\left(E-\mu_{k}\right)} Q(E)
$$

where $Q(E)=\prod_{k=1}^{m-1}\left(E-\mu_{k}\right)$. By setting $E=\mu_{k}$, we have

$$
b_{k}^{2}=-\frac{\prod_{j=1}^{m}\left(\mu_{k}-\nu_{j}\right)}{\prod_{j \neq k}^{m-1}\left(\mu_{k}-\mu_{j}\right)} \geq 0
$$


where the negative sign here is because of the interlacing property $\nu_{k}>\mu_{k}$. One can solve for $\omega_{m}$ recursively using Eq. (14) and (15). We found a recursive relation $\omega_{m}^{2}=\bar{\omega}_{m-1}^{2}+\frac{1}{2} \Delta_{N-1}\left(\sum_{k=1}^{(N-1) / 2} \Delta_{2 k-1}\right)$, where $\bar{\omega}_{m-1}$ is the counterpart of $\omega_{m}$ when we solve the same inverse eigenvalue problem without the last two eigenvalues $E_{N-1}$ and $E_{N}$. Since $\omega_{m}^{2}$ is a positive sum of the products $\Delta_{j} \Delta_{k}$, it is minimized when all $\Delta_{k}=\tilde{\Delta}_{\text {min }}$, and hence $\omega_{\max } \geq \omega_{m} \geq \tilde{\omega}_{\max }$. This completes our proof for $\eta \geq 1$ for all possible engineered spin chains.

In the following, we shall analyze the implications of the speed limit. It is natural to ask, apart from the advantage of not requiring dynamical controls, if the schemes of engineered chains can be fundamentally faster than the series of swaps (SOS) protocol mentioned at the beginning. The answer is positive. Suppose we now consider the ideal case where each swap operation can be achieved with the same maximum possible coupling $\tilde{\omega}_{\max }$ in Eq. (8), the evolution time for each swap is $\tau_{0}=\pi / 2 \tilde{\omega}_{\max }$. Since it will totally take $N-1$ steps to transfer a state through a chain of $N$ spins, we can generalize our definition of the efficiency for this case as: $\eta_{\text {sos }}=(N-1) / 2 \tilde{\omega}_{\max }$, which is an increasing function of $N$, and one can easily show that $1 \leq \eta_{\text {sos }}<2$ for $N \geq 2$. Therefore, the engineered spin chains can have roughly a factor of two gain in speed for sufficiently long chains.

According to the Margolus-Levitin theorem [6], the minimum time for a quantum state $\left|\psi_{i}\right\rangle$ to evolve into an orthogonal state $\left|\psi_{f}\right\rangle$, where $\left\langle\psi_{f} \mid \psi_{i}\right\rangle=0$, is limited by the initial energy: $\max \left(\frac{\pi}{2\left(E-E_{0}\right)}, \frac{\pi}{2 \Delta E}\right)$, where $E=\left\langle\psi_{i}|H| \psi_{i}\right\rangle$ is the initial energy, $E_{0}$ is the ground state energy and $\Delta E=\sqrt{\left\langle\psi_{i}\left|(H-E)^{2}\right| \psi_{i}\right\rangle}$ is the energy uncertainty of the initial state. The computational power of physical systems as quantum computers was analyzed [7] along this line of thought. Here we shall argue that, at least for solid state systems where the interactions are local, it is more realistic to characterize the computational power by the couplings in the Hamiltonian than the initial energy. We shall again focus on the 1D QST problem [cf. Eq.(2)] as an example, as the generalization to more complicated quantum operations is straightforward through the scheme proposed in Ref. [11]. For the linear spectrum, we have $E-E_{0}=N / 2$ for large $N$. Given the same amount of initial energy $N / 2$, the minimal evolution time for the SOS protocol is $\tau=\pi / N$ (as $E-E_{0}=\Delta E$ ). For a linear chain of $N$ spins, the SOS protocol seems to be as efficient as the engineered chains. However, as we have just analyzed, for each swap the tunneling strength would be $N / 2$, which is twice of the maximum possible coupling $\tilde{\omega}_{\max }$ assumed before!

In conclusion, we have generalized the study of quantum speed limit to the problem of quantum state transfer in one dimension. We have argued that for solid state systems it is more realistic to characterize the computation power explicitly by the coupling terms in the Hamiltonian than the initial energy. This work also implies that quantum algorithms, when implemented in solid state devices, can be optimized, if we employ the concept of spin chain engineering 11]. More practically, systematic errors are expected to be significantly minimized with the reduction of dynamical controls. We lastly comment that such speed limit, if not confined with nearest-neighbor interactions in one dimension, could be overcome by increasing either the network complexity [9] or the Hamiltonian complexity [26]. Details to be discussed elsewhere.

MHY acknowledges the support of the NSF grant DMR-03-50842 and the Croucher Foundation and thanks S. C. Benjamin, S. Bose, Alec Maassen van den Brink and L. Maccone for valuable discussions.

* Electronic address: myung2@uiuc.edu

[1] D. Bouwmeester, A. Ekert, and A. Zeilinger, The Physics of Quantum Information (Springer-Verlag, Berlin, 2000).

[2] D. P. DiVincenzo, Fortschr. Phys. 48, 9 (2000).

[3] J. I. Cirac, P. Zoller, H. J. Kimble, and H. Mabuchi, Phys. Rev. Lett. 78, 3221 (1997).

[4] B. E. Kane, Nature (London) 393, 133 (1998).

[5] In many systems, even though the two-body interactions are intrinsically not switchable, their effects are usually made to be annulled by some refocusing techniques.

[6] N. Margolus and L. B. Levitin, Physica D 120, 188 (1998); P. Kosiński and M. Zych, Phys. Rev. A 73, 024303 (2006).

[7] S. Lloyd, Nature (London) 406, 1047 (2000).

[8] S. Bose, Phys. Rev. Lett. 91, 207901 (2003).

[9] M.-H. Yung, D. W. Leung, and S. Bose, Quantum Inf. Commun. 4, 174 (2003).

[10] M.-H. Yung and S. Bose, Phys. Rev. A 71, 032310 (2005).

[11] M.-H. Yung, S. C. Benjamin and S. Bose, e-print quant-ph/0508165 (To appear in Physical Review Letter).

[12] M. Christandl, N. Datta, A. Ekert and A. J. Landahl, Phys. Rev. Lett. 92, 187902 (2004); M. Christandl, N. Datta, T. C. Dorlas, A. Ekert, A. Kay, and A. J. Landahl, Phys. Rev. A 71, 032312 (2005).

[13] C. Albanese, M. Christandl, N. Datta and A. Ekert, Phys. Rev. Lett. 93, 230502 (2004).

[14] T. S. Cubitt, F. Verstraete, and J. I. Cirac, Phys. Rev. A 71, 052308 (2005).

[15] T. Shi, Y. Li, Z. Song, and C. P. Sun, Phys. Rev. A 71, 032309 (2005).

[16] S. Yang, Z. Song and C. P. Sun, e-print quant-ph/0510109

[17] A. Kay and M. Ericsson, New J. Phys. 7, 143 (2005).

[18] A. Kay, Phys. Rev. A 73, 032306 (2006).

[19] S. R. Clark, C. Moura Alves and D. Jaksch, New J. Phys. 7, 124 (2005).

[20] P. Karbach and J. Stolze, Phys. Rev. A 72, 030301(R) (2005).

[21] G. De Chiara, D. Rossini, S. Montangero and R. Fazio, Phys. Rev. A 72, 012323 (2005).

[22] As pointed out in Ref. [17], the same set of coupling was first proposed by Cook and Shore in 1979 [R. J. Cook 
and B. W. Shore, Phys. Rev. A 20, 539 (1979)] in the context of coherent population transfer between different atomic levels.

[23] Our result on the quantum speed limit is not necessarily confined by the exact form of this Hamiltonian. In fact, it is generally applicable to many hopping processes of spins or particles, as long as the effective Hamiltonian is of the form described in Eq. (3).
[24] D. Boley and G. H. Golub, Inverse Problems 3, 595 (1987).

[25] G. M. L. Gladwell, Inverse problems in vibration (Kluwer Academic, Boston, 1986).

[26] V. Giovannetti, S. Lloyd, L. Maccone, Europhys. Lett. 62, 615 (2003). 